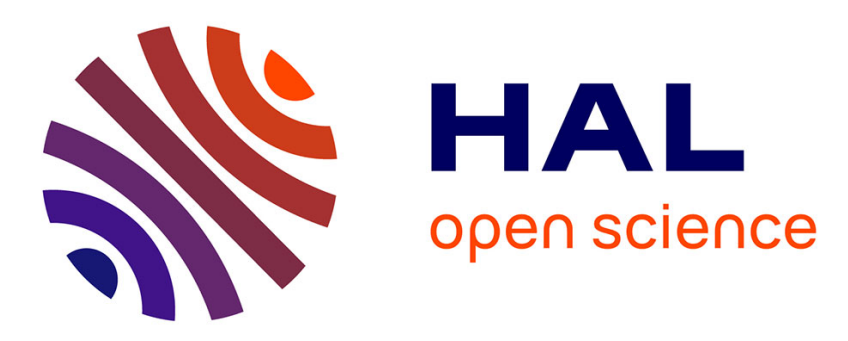

\title{
Analysis of the propagation of plane acoustic waves in passive periodic materials using the finite element method
}

Philippe Langlet, Anne-Christine Hladky, Jean-noël Decarpigny

\section{- To cite this version:}

Philippe Langlet, Anne-Christine Hladky, Jean-noël Decarpigny. Analysis of the propagation of plane acoustic waves in passive periodic materials using the finite element method. Journal of the Acoustical Society of America, 1995, 98 (5), pp.2792-2800. 10.1121/1.413244 . hal-03300156

\section{HAL Id: hal-03300156 https://hal.science/hal-03300156}

Submitted on 26 Jul 2021

HAL is a multi-disciplinary open access archive for the deposit and dissemination of scientific research documents, whether they are published or not. The documents may come from teaching and research institutions in France or abroad, or from public or private research centers.
L'archive ouverte pluridisciplinaire HAL, est destinée au dépôt et à la diffusion de documents scientifiques de niveau recherche, publiés ou non, émanant des établissements d'enseignement et de recherche français ou étrangers, des laboratoires publics ou privés. 


\title{
Analysis of the propagation of plane acoustic waves in passive periodic materials using the finite element method
}

\author{
Philippe Langlet, Anne-Christine Hladky-Hennion, and Jean-Noël Decarpigny \\ IEMN (UMR 9929 CNRS), ISEN Department, Institut Supérieur d'Electronique du Nord, 41 boulevard \\ Vauban, 59046 Lille Cedex, France
}

(Received 30 September 1994; revised 19 May 1995; accepted 29 May 1995)

\begin{abstract}
The finite element approach has been previously used, with the help of the ATILA code, to model the scattering of acoustic waves by single or doubly periodic passive structures [A. C. Hladky-Hennion et al., J. Acoust. Soc. Am. 90, 3356-3367 (1991)]. This paper presents a new extension of this technique to the analysis of the propagation of plane acoustic waves in passive periodic materials without losses and describes with particular emphasis its application to doubly periodic materials containing different types of inclusions. In the proposed approach, only the unit cell of the periodic material has to be meshed, thanks to Bloch-Floquet relations. The modeling of these materials provides dispersion curves from which results of physical interest can be easily extracted: identification of propagation modes, cutoff frequencies, passbands, stopbands, as well as effective homogeneous properties. In this paper, the general method is first described, and particularly the aspects related to the periodicity. Then a test example is given for which analytical results exist. This example is followed by detailed presentations of finite element results, in the case of periodic materials containing inclusions or cylindrical pores. The homogenized properties of porous materials are determined with the help of an anisotropic model, in the large wavelength limit. A validation has been carried out with periodically perforated plates, the resonance frequencies of which have been measured. The efficiency and the versatility of the method is thus clearly demonstrated. (c) 1995 Acoustical Society of America.
\end{abstract}

PACS numbers: 43.20.Bi, 43.20.Gp, 43.20.Jr, 43.35.Cg

\section{INTRODUCTION}

Periodic materials, such as porous or fibrous materials and composites, have araisen a great deal of interest and are now widely used in underwater acoustics, signal processing, as well as for medical imaging applications. Particularly, in order to explain their physical behavior, the propagation of harmonic elastic waves through periodic materials can be studied. From the dispersion curves, many results of physical interest can be easily extracted: propagation modes, cutoff frequencies, passbands, stopbands, as well as effective homogeneous properties of these structures and their validity limits.

Many authors have built accurate mathematical models for the propagation of harmonic elastic waves through periodic materials, based on the Bloch-Floquet theory. ${ }^{1}$ Thus Audoly and Duméry have studied periodic materials containing cylindrical inclusions, using a multiple-scattering approach. ${ }^{2}$ Tao and Sheng used a method based on the iterative solution of the inhomogeneous elastic wave equation in the case of porous materials, ${ }^{3}$ whereas Auld et al. developed an elastodynamic theory for periodic composite materials. ${ }^{4}$ Braga and Herrmann ${ }^{5}$ have studied acoustic propagation through periodically layered composites. Nemat-Nasser and Yamada $^{6}$ and Baste and Gérard ${ }^{7}$ have analyzed the propagation of harmonic elastic waves through one-directional or three-directional composites. Though efficient, all these methods require in many cases a lot of algebraic developments, which restrict their use to a small number of given geometries or to given propagation directions.
To avoid model restriction, several authors have also used a numerical approach. Using the boundary integral equation method, Achenbach and Kitahara ${ }^{8}$ have provided dispersion curves for a periodic material containing spherical cavities. Bennet ${ }^{9}$ has used a transfer-matrix approach coupled to the finite element method to describe the dispersion curves in a periodic material: this method has provided good results but is restricted to propagation directions parallel to the periodicity. Ruffa ${ }^{10}$ has used a three-dimensional finite element analysis to determine the acoustic behavior of plane waves propagating through bubbly liquids having periodic bubble distributions. Also, Ma et al. ${ }^{11}$ have developed a finite element method in the case of infinitely long, cylindrical pores when the waves propagate perpendicularly to the pores axis. This method uses the symmetry of the elementary periodic cell. The finite element approach has been previously used by Hladky-Hennion et al., ${ }^{12-14}$ with the help of the ATILA code, ${ }^{15-19}$ to model the scattering of acoustic waves by single periodic passive structures, such as compliant tube gratings, ${ }^{12}$ by doubly periodic passive structures, such as Alberich anechoic coatings, ${ }^{13}$ or to study the freefield voltage sensitivity and the transmitting voltage response of active periodic structures, such as 1-3 piezocomposites. ${ }^{14}$ The efficiency and the versatility of the finite element method to describe the acoustical behavior of periodic structures have been demonstrated, particularly because this approach allows the modeling of any geometrical shapes, made up with any elastic or piezoelectric, isotropic or anisotropic media, by simply building the specific mesh. The aim of this paper is to extend the finite element approach previously 
described ${ }^{12-14}$ to the study of the propagation of harmonic elastic waves in passive periodic materials. ${ }^{20}$

In the method proposed here, the periodic material is supposed to be periodic in one, two, or three space directions, finite or infinite in the others. Because of BlochFloquet relations between displacement values at points which are separated by the geometrical period, only one unit cell is meshed with finite elements. ${ }^{12-14}$ Within this cell, a phase relation is applied on nodes separated by one period, defining boundary conditions between adjacent cells. The phase relation is related to the wave number of the incident wave in the periodic material. The dispersion curves present the variations of the eigenfrequencies versus the wave number. They provide phase velocity and group velocity for each propagation mode, stopbands, passbands, etc.

In the first part of this paper, the theoretical formulation is given, with emphasis on the aspects related to the periodicity. For the sake of simplicity, only the two-dimensional mathematical model is described. In the second part, the comparison between numerical and analytical results obtained for an homogeneous and isotropic material is first presented and displays a nice agreement validating the method. Then the results obtained for a periodic array of square inclusions embedded in a matrix are presented. Finally, materials containing a periodic array of cylindrical pores are studied. The dispersion curves obtained by calculation provide phase velocity, in the large wavelength limit. So, the homogenized properties are determined with the help of an anisotropic model. Using these effective properties, the resonance frequencies of perforated plates are determined with the help of the finite element method and are compared to the measured resonance frequencies. Then, the homogenization process is validated.

\section{THEORETICAL FORMULATION}

\section{A. General mathematical model}

To present the model, although a lot of other cases can also be dealt with, the doubly periodic material of reference is an infinite elastic medium with a periodic array of cylindrical pores which is described in Fig. 1. The material is without losses. In this model, the pores are infinite and are set parallel to the $z$ axis. Thus the problem is bidimensional and only depends on the $x$ and $y$ coordinates, using plane strain conditions.

The material is theoretically assumed to be infinite in the $x$ and $y$ directions, and to be doubly periodic. The whole domain is split into successive unit cells. Each unit cell contains one cylindrical pore and a small part of the surrounding material. This cell is detailed in Fig. 2. Due to the periodicity of the material, the $A_{1}$ and $A_{2}$ lines, parallel to the $y$ axis, and the $B_{1}$ and $B_{2}$ lines, parallel to the $x$ axis, limit the unit cell, which is $2 d_{a}$ wide in the $x$ direction and $2 d_{b}$ wide in the $y$ direction. In Fig. 2, corners are marked by the letter $\mathrm{C}$.

Then the material is excited by a plane, monochromatic wave, the direction of incidence of which is marked by an angle $\theta$ with respect to the positive $y$ axis. The incident wave is characterized by a real wave vector $\mathbf{k}$, the modulus of

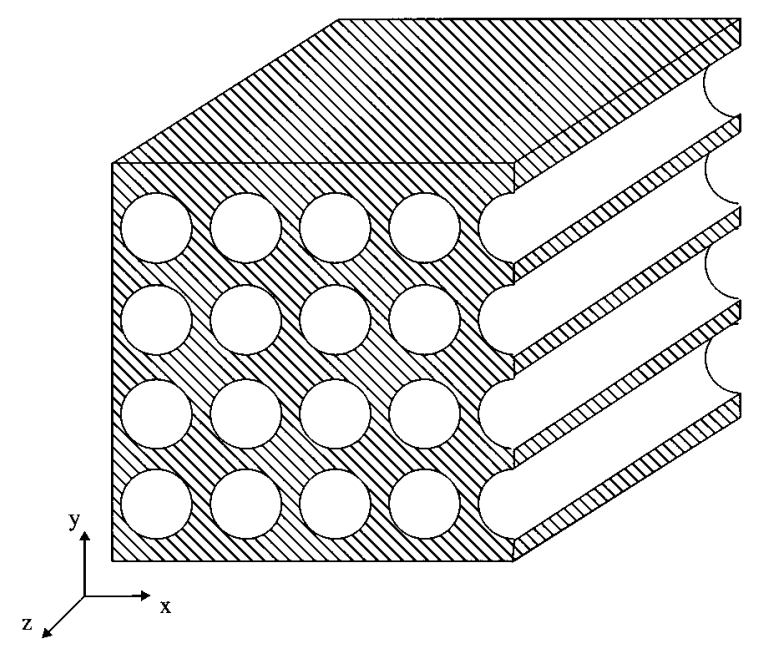

FIG. 1. Schematic description of a doubly periodic material.

which is called the wave number and is denoted $k$. The time dependence is written as $e^{-j \omega t}$.

Because the material is assumed to be periodic and infinite in the $x$ and $y$ directions, any space function $F$ (displacement, ...) has to verify the classical Bloch relation

$$
\begin{aligned}
F\left(x+2 d_{a}, y+2 d_{b}\right) & =e^{j 2 d_{a} k \sin \theta} e^{j 2 d_{b} k \cos \theta} F(x, y) \\
& =e^{j \varphi_{a}} e^{j \varphi_{b}} F(x, y) .
\end{aligned}
$$

\section{B. Finite element modeling of the unit cell}

Using relation (1) allows us to reduce the model to only one unit cell, which can be meshed using finite elements. ${ }^{21}$ Writing relation (1) between the displacement values for nodes that are separated by one period provides the boundary conditions between adjacent cells. Using the finite element method with the help of the ATILA code, ${ }^{12-19}$ the unit cell is meshed and divided into elements connected by nodes. Fig-

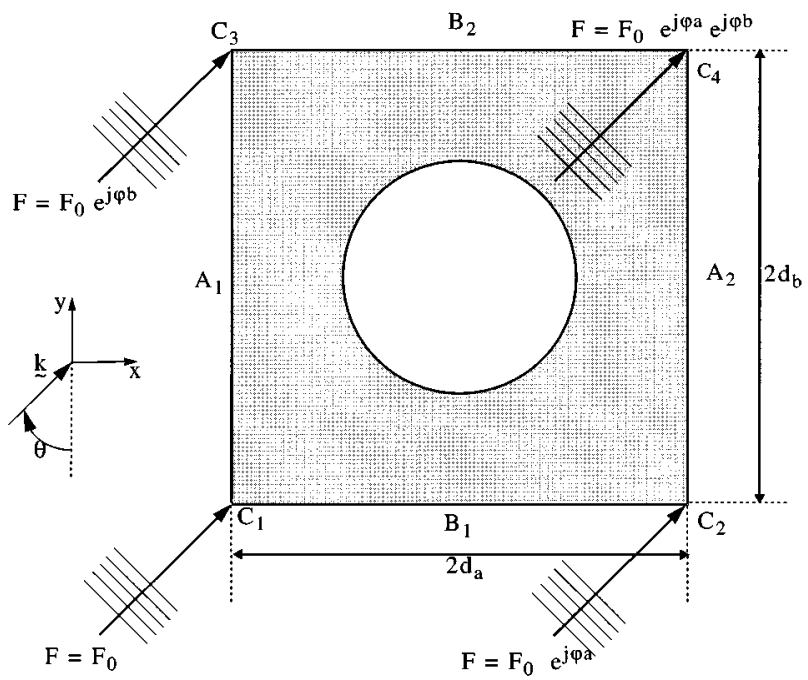

FIG. 2. Schematic description of a doubly periodic material, used to define the $A_{1}, A_{2}, B_{1}, B_{2}$ lines, the $C_{1}, C_{2}, C_{3}, C_{4}$ points, and the incident wave. 


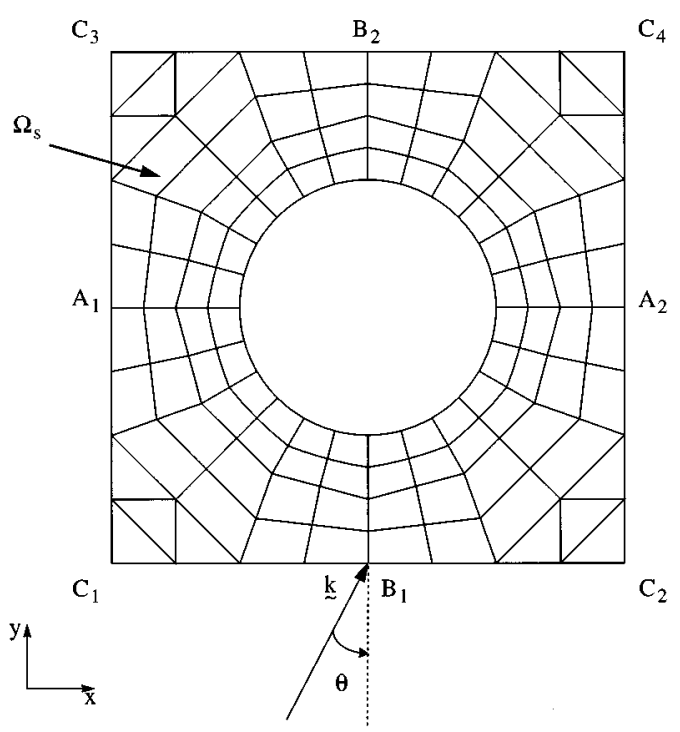

FIG. 3. Finite element mesh of the unit cell.

ure 3 presents the unit cell, containing the solid structure $\Omega_{s}$, the boundary lines $A_{1}, A_{2}, B_{1}$, and $B_{2}$, and the corners $C_{1}, C_{2}, C_{3}$, and $C_{4}$.

If a modal analysis is considered, the whole system of equations is, classically

$$
\left(\left[K_{u u}\right]-\omega^{2}[M]\right) \mathbf{U}=\mathbf{F},
$$

where the unknown is the vector of nodal values of the displacement $\mathbf{U} .\left[K_{u u}\right]$ and $[M]$ are, respectively, the structure stiffness and coherent mass matrices. $\omega$ is the angular frequency. $\mathbf{F}$ contains the nodal values of the applied forces. $\left[K_{u u}\right],[M]$, and $\mathbf{F}$ result, respectively, from the merging of elementary matrices and vector $\left[K_{u u}^{e}\right],\left[M^{e}\right]$, and $\mathbf{F}^{e}$, where $e$ stands for the element number. In all the following examples, isoparametric elements are used, with a quadratic interpolation along element sides.

\section{Application of the periodic boundary conditions}

The application of the periodic boundary conditions implies that the phase relation (1) between nodal values belonging to the $A_{1}$ and $A_{2}$ lines, on the one hand, to the $B_{1}$ and $B_{2}$ lines, on the other hand, has to be incorporated in the matrix equation (2). The unit cell is divided into nine parts: the four lines $A_{1}, A_{2}, B_{1}$, and $B_{2}$, the four corners $C_{1}, C_{2}, C_{3}$, and $C_{4}$, and the inner domain $I$. Displacement vector $\mathbf{U}$ and force vector $\mathbf{F}$ are then split into the corresponding nine parts. Due to relation (1), their components have to verify

$$
\begin{array}{ll}
\mathbf{U}_{A 2}=\mathbf{U}_{A 1} e^{j \varphi_{a}}, & \mathbf{U}_{B 2}=\mathbf{U}_{B 1} e^{j \varphi_{b}}, \quad \mathbf{U}_{C 2}=\mathbf{U}_{C 1} e^{j \varphi_{a}}, \\
\mathbf{U}_{C 3}=\mathbf{U}_{C 1} e^{j \varphi_{b}}, & \mathbf{U}_{C 4}=\mathbf{U}_{C 1} e^{j\left(\varphi_{a}+\varphi_{b}\right)} .
\end{array}
$$

Then, owing to the equilibrium of interconnecting forces between two adjacent cells, relation (1) leads to analogous relations for the force vector. $\mathbf{F}_{I}$, which corresponds to forces applied to inner nodes, is equal to zero. Defining the reduced vector $\mathbf{U}_{R}$ as a vector containing the nodal values of the displacement on the $A_{1}$ and $B_{1}$ lines, on the $C_{1}$ corner and in the inner domain $I$, relations (3) imply a simple matrix relation between $\mathbf{U}$ and $\mathbf{U}_{R}$ which can be written

$$
\mathbf{U}=\left[P_{U}\right] \mathbf{U}_{R}=\left[P_{U}\right]\left[\begin{array}{c}
\mathbf{U}_{A 1} \\
\mathbf{U}_{B 1} \\
\mathbf{U}_{C 1} \\
\mathbf{U}_{I}
\end{array}\right] .
$$

In the same way, a matrix relation can be defined between the vector $\mathbf{F}$ and the reduced vector $\mathbf{F}_{R}$ :

$$
\mathbf{F}=\left[P_{F}\right] \mathbf{F}_{R}=\left[P_{F}\right]\left[\begin{array}{c}
\mathbf{F}_{A 1} \\
\mathbf{F}_{B 1} \\
\mathbf{F}_{C 1} \\
\mathbf{0}
\end{array}\right] .
$$

Thus the equation to be solved can be reduced to

$$
\begin{aligned}
{\left[P_{U}\right]^{* T}\left(\left[K_{u u}\right]-\omega^{2}[M]\right)\left[P_{U}\right] \mathbf{U}_{R} } & =\left(\left[K_{R}\right]-\omega^{2}\left[M_{R}\right]\right) \mathbf{U}_{R} \\
& =\left[P_{U}\right]^{* T}\left[P_{F}\right] \mathbf{F}_{R} .
\end{aligned}
$$

Finally, the matrices $\left[K_{R}\right]$ and $\left[M_{R}\right]$ are divided following four parts, $A_{1}, B_{1}, C_{1}$ and $I$, and the resulting equation is

$$
\left(\left[K_{R}\right]-\omega^{2}\left[M_{R}\right]\right) \mathbf{U}_{R}=\mathbf{0},
$$

where

$$
\begin{aligned}
& {\left[K_{R}\right]=\left[P_{U}\right]^{* T}\left[K_{u u}\right]\left[P_{U}\right],} \\
& {\left[M_{R}\right]=\left[P_{U}\right]^{* T}[M]\left[P_{U}\right] .}
\end{aligned}
$$

The detailed expressions of $\left[K_{R}\right]$ and $\left[M_{R}\right]$ are presented in Appendix A of Ref. 13. In terms of finite elements, this operation is the static condensation of degrees of freedom belonging to $A_{2}, B_{2}, C_{2}, C_{3}$, and $C_{4}$. The reduced matrices $\left[K_{R}\right]$ and $\left[M_{R}\right]$ are Hermitian, because $\omega$ eigenvalues and $k$ wave numbers are real. The computation of $\omega$ eigenvalues when the wave vector is complex necessitates the development of new algorithms, which are not yet available.

The angular frequency $\omega$ is a periodical function of the wave vector $\mathbf{k}$. Thus the problem can be reduced to the first Brillouin zone. ${ }^{1}$ The dispersion curves are built varying $\mathbf{k}$ on the first Brillouin zone, for a given propagation direction. The whole diagram is deduced using symmetries. In the case of Fig. 2, the wave number $k$ varies as

$$
\begin{aligned}
& \theta_{0}=\operatorname{atan}\left(d_{b} / d_{a}\right), \\
& 0 \leqslant|\theta| \leqslant \theta_{0} \Rightarrow 0 \leqslant k \leqslant \frac{\pi}{2 d_{b} \cos \theta}, \\
& \theta_{0} \leqslant|\theta| \leqslant \frac{\pi}{2} \Rightarrow 0 \leqslant k \leqslant \frac{\pi}{2 d_{a} \sin \theta} .
\end{aligned}
$$

\section{RESULTS}

\section{A. Homogeneous and isotropic material}

In this section, the propagation of plane harmonic waves in an infinite, homogeneous, and isotropic elastic medium is studied as a classical test example. The material is aluminum, whose physical properties are $E=7.31 \times 10^{10} \mathrm{~Pa}, \nu$ $=0.325$, and $\rho=2770 \mathrm{~kg} \mathrm{~m}^{-3}$. Using plane strain conditions, 


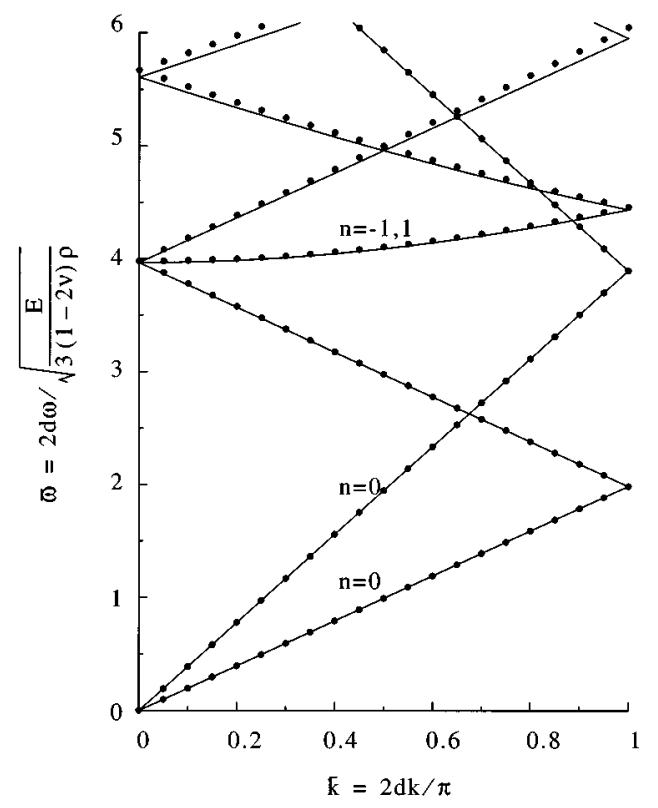

FIG. 4. Dispersion curves for a homogeneous, isotropic material: Variations of the reduced angular frequencies $\bar{\omega}$ as a function of the reduced wave number $\bar{k}$ Full lines: analytical results. Markers: FEM results.

the material is supposed to be infinite in the $z$ direction and is set in the $x y$ plane. Using the finite element method to solve this problem, an arbitrary square unit cell is meshed, whose side $2 d$ is equal to $4 \mathrm{~cm}$. It is partitioned into 16 finite elements. The wave vector $\mathbf{k}$ is selected in the first Brillouin zone in the $x y$ plane.

The calculation is performed when $\mathbf{k}$ is chosen parallel to the $y$ axis, which corresponds to a normal incident wave vector $\left(\theta=0^{\circ}\right)$. Figure 4 presents the variations of the reduced eigenangular frequencies $[\bar{\omega}=\omega 2 d \sqrt{E / 3(1-2 \nu)}]$ as a function of the reduced wave number $(\bar{k}=k 2 d / \pi)$. The finite element results are compared to the analytical results, following the relation

$$
\omega=c \sqrt{(n \pi / d)^{2}+\left(k_{y}+m \pi / d\right)^{2}},
$$

where $n$ and $m$ are integers, and $c$ is either equal to the longitudinal wave velocity $c_{L}$ or to the transverse wave velocity $c_{T}$. When $n$ is not equal to zero, the corresponding branches are hyperbolic curves and are due to the wave vector whose components are determined as modulus $2 \pi$ and could be set in any Brillouin zone. ${ }^{1}$

The agreement between the curves is quite good, when the reduced angular frequency $\bar{\omega}$ is lower than 4 . It corresponds to the validity limit of the $\lambda / 4$ criterion, ${ }^{22}$ which states that the largest length of each element in a given mesh has to be smaller than a quarter of the transverse wavelength in the material. This section is a strong validation for the method.

Using a nonsymmetrical mesh instead of a symmetrical mesh gives identical results. ${ }^{20}$ When $\mathbf{k}$ is not parallel to the $x$ or $y$ axis, the agreement between the finite element results and the analytical results is perfect. In this case the analytical relation is similar to relation (10), but $k_{x}$ is not equal to 0 . A few examples are detailed in Ref. 20.

\section{B. Periodic array of square inclusions}

In this case, the periodic material is a periodic array of square inclusions, supposed to be infinite in one direction. Then the problem is bidimensional. The square inclusions, the side of which is equal to $2 a$, are made of brass ( $E$ $=9.2 \times 10^{10} \mathrm{~Pa}, \nu=0.33, \rho=8270 \mathrm{~kg} \mathrm{~m}^{-3}$ ), denoted material 1 , characterized by its longitudinal velocity $c_{L 1}$ and transverse velocity $c_{T 1}$. They are embedded in aluminum, denoted material 2 , characterized by its longitudinal velocity $c_{L 2}$ and transverse velocity $c_{T 2}$. They are arranged as a doubly periodic material. The grating spacing is denoted $2 d$ (Fig. 5). For a)

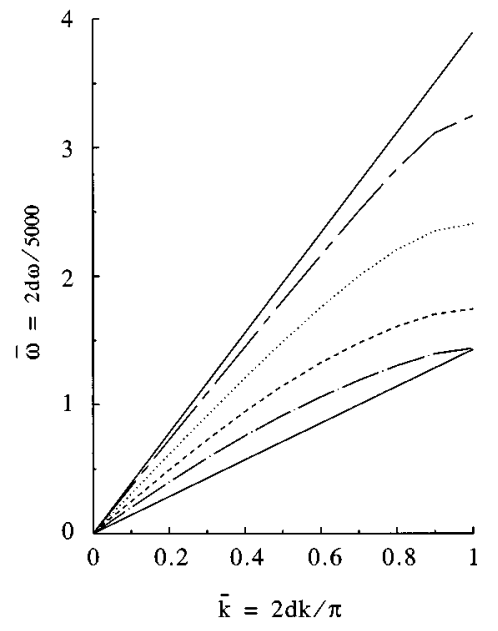

b)

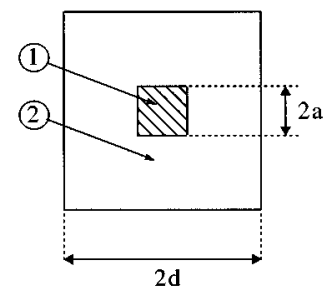

$\begin{array}{r}\mathrm{a} / \mathrm{d}=0.00 \\ \mathrm{a} / \mathrm{d}=0.25 \\ \hline \mathrm{a} / \mathrm{d}=0.50 \\ \mathrm{a} / \mathrm{d}=0.75 \\ \mathrm{a} / \mathrm{d}=0.90 \\ \mathrm{a} / \mathrm{d}=1.00 \\ \hline\end{array}$

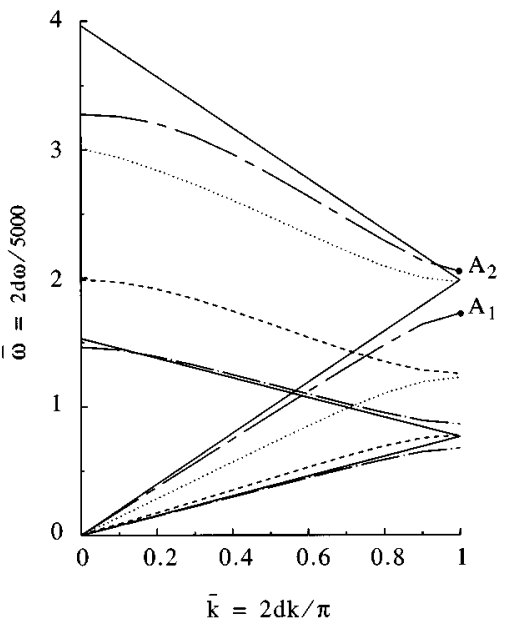

FIG. 5. Dispersion curves for a periodic array of square inclusions for different ratios $a / d$ : Variations of the reduced angular frequencies $\bar{\omega}$ as a function of the reduced wave number $\bar{k}$ (a) First longitudinal mode. (b) First two transverse modes. 


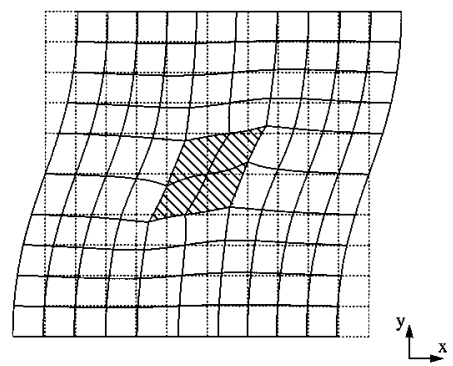

$\mathrm{A}_{2}$

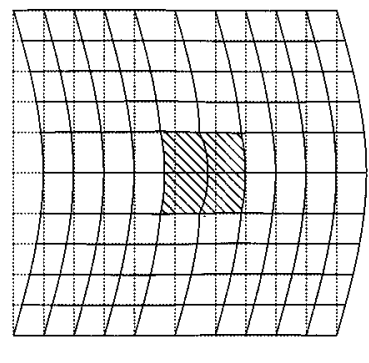

FIG. 6. Periodic array of square inclusions: Views of the displacement field at given instant, for $a / d=0.25$ with $k=\pi / 2 d$. (a) First mode (point $A_{1}$ ). (b) Second mode (point $A_{2}$ ).

this analysis, the wave vector $\mathbf{k}$ is set parallel to the $y$ axis.

Figure 5(a) presents the dispersion curves for the first longitudinal mode when the ratio $a / d$ is varying from 0 to 1 . Clearly, the quasilongitudinal velocity, in the large wavelength limit, varies from $c_{L 2}$ to $c_{L 1}$. In the same way, Fig. 5(b) presents the dispersion curves for the first and second transverse modes. The quasitransverse velocity varies from $c_{T 2}$ to $c_{T 1}$. Moreover, the introduction of inhomogeneities in the unit cell implies the opening of a gap at the Brillouin zone boundary. The passbands are related to real wave numbers and stopbands are related to complex wave numbers (evanescent waves), which are not calculated and do not appear in Fig. 5. The largest stopband is when $a / d=0.5$. This fact can be easily explained using a view of displacement fields at a given instant for the first transverse mode at the boundary of the Brillouin zone (Fig. 6). In the heterogeneous medium, these displacement fields are different, due to the inclusion. Thus the corresponding eigenvalues are different. However, in an homogeneous medium, these displacement fields become identical, considering a translation of length $d$ along the wave vector $\mathbf{k}$.

\section{Periodic array of cylindrical pores}

A periodic material containing infinite cylindrical pores with circular cross section is considered. The pores are periodically spaced in the $x$ and $y$ directions. Air cavities are not meshed. The material is PVC $\left(E=3.78 \times 10^{9} \mathrm{~Pa}, \nu=0.4\right.$, $\rho=1430 \mathrm{~kg} \mathrm{~m}^{-3}$ ) in which longitudinal and transverse velocities are denoted $c_{L}$ and $c_{T}$. The radius of the pore is denoted $a$. The porosity is defined as $P=\pi a^{2} / 4 d^{2}$, where $2 d$ is the pore spacing. The maximum value for $P$ is $\pi / 4$, which corresponds to a contact between the pores. The wave vector $\mathbf{k}$ is set perpendicular to the pore axis, and parallel to the $y$ axis. Figure 7 presents the dispersion curves for $P$ $=0.503 \mathrm{a} / \mathrm{d}=0.8$ ). Because the unit cell is symmetric with respect to the $y$ axis and because the wave vector is parallel to this axis, the curves can be split into symmetrical or antisymmetrical branches, which are identified with the help of the computed displacement field. The full lines correspond to symmetrical modes with respect to the $y$ axis. The first one is a quasilongitudinal propagative mode. The dashed lines cor-
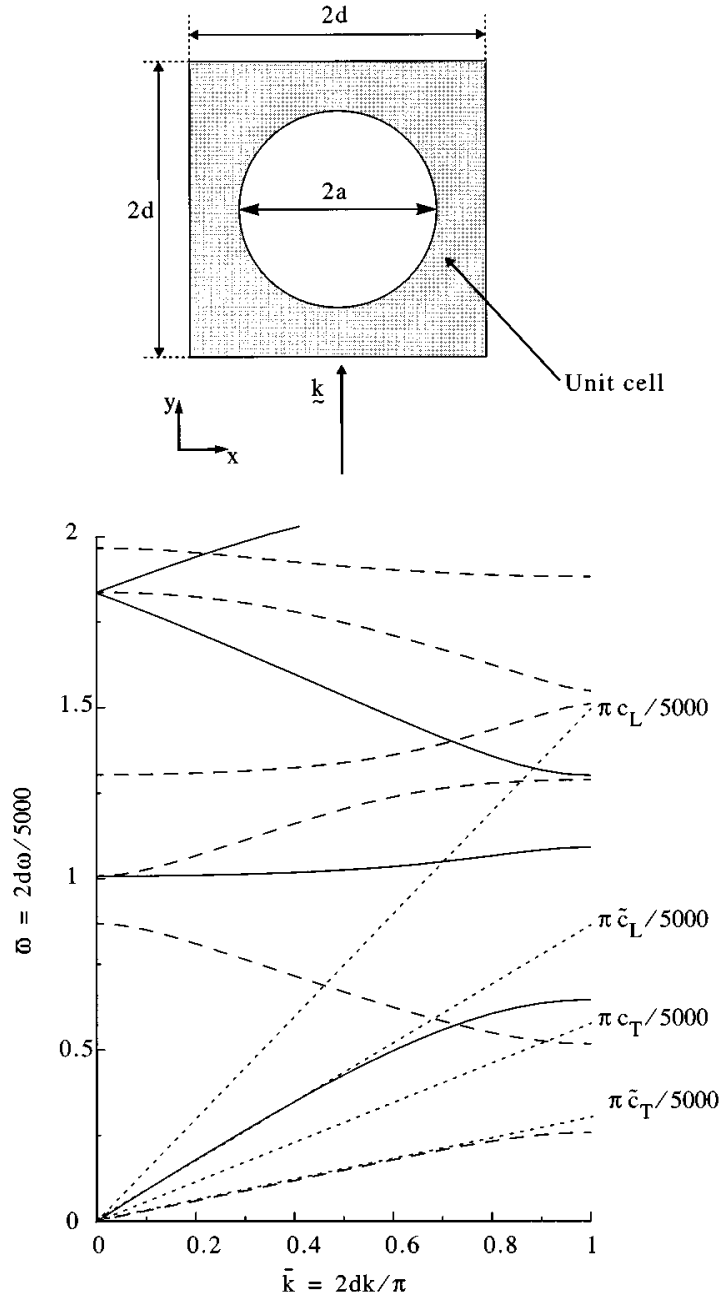

FIG. 7. Dispersion curves for a periodic array of cylindrical pores with $P$ $=0.503$ : Variations of the reduced angular frequencies $\bar{\omega}$ as a function of the reduced wave number $\bar{k}$ Full lines: symmetrical modes with respect to the $y$ axis. Dashed lines: antisymmetrical modes with respect to the $y$ axis. Dotted lines: lines which slopes correspond to the longitudinal or transverse velocities in the homogeneous material $\left(c_{L}, c_{T}\right)$ and in the homogenized material in the large wavelength limit $\left(\tilde{c}_{L}, \tilde{c}_{T}\right)$.

respond to antisymmetrical modes. The first one is a quasitransverse propagative mode. There is no crossing between the branches of one family (symmetrical or antisymmetrical), whereas symmetrical branches can cross antisymmetrical branches. The introduction of pores in the material induces the opening of a gap at the Brillouin zone boundary.

The low-frequency branches of the curves can be approximated to two straight lines whose slopes are $\pi \tilde{c}_{L} / 5000$ and $\pi \tilde{c}_{T} / 5000$. They can be compared to the dispersion curves for PVC without inclusions, which are straight lines of slope $\pi c_{L} / 5000$ and $\pi c_{T} / 5000$. In that case, the sound propagates slower in the heterogeneous medium than in the homogeneous medium, and the effect increases with porosity.

For different porosities, the dispersion curves have been drawn and the phase velocities in the large wavelength limit have been calculated, using the slope of the low-frequency branches. Figure 8 presents the variations of the normalized longitudinal and transverse velocities $\tilde{c}_{L} / c_{L}$ and $\tilde{c}_{T} / c_{T}$ as a 


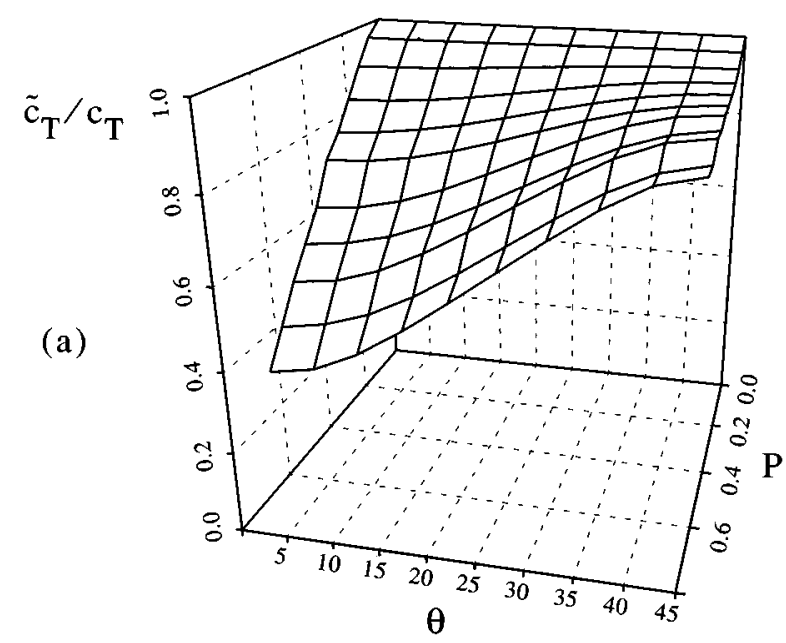

$\tilde{\mathrm{c}}_{\mathrm{L}} / \mathrm{c}_{\mathrm{L}}$

(b)

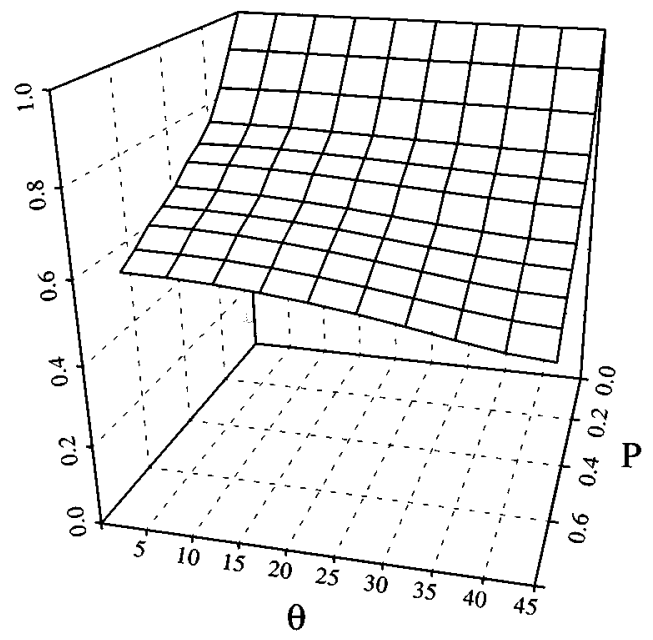

FIG. 8. Variations of the normalized longitudinal velocity $\tilde{c}_{L} / c_{L}$ and of the normalized transverse velocity $\tilde{c}_{T} / c_{T}$ as a function of the porosity $P$ and of the incident angle $\theta$, in the case of a periodic array of cylindrical pores.

function of the porosity $P$ and of the incident angle $\theta$. As the porosity increases, the wave velocities decrease.

For a given porosity, the quasitransverse velocity $\tilde{c}_{T}$ and the quasilongitudinal velocity $\tilde{c}_{L}$ vary as a function of the incident angle. So, the porous material must be considered as an homogeneous anisotropic medium, in the large wavelength limit. The effective density $\tilde{\rho}$ verifies the relation

$$
\tilde{\rho}=(1-P) \rho .
$$

The effective stiffness tensor $[\tilde{c}]$ is chosen as

$$
[\tilde{c}]=\left[\begin{array}{ccc}
\tilde{c}_{11} & \tilde{c}_{12} & 0 \\
\tilde{c}_{12} & \tilde{c}_{11} & 0 \\
0 & 0 & \tilde{c}_{66}
\end{array}\right] .
$$

Using the finite element method, the computation of velocities in the large wavelength limit has been performed for only two incident angles $\theta$. Thanks to Christoffel's equations,

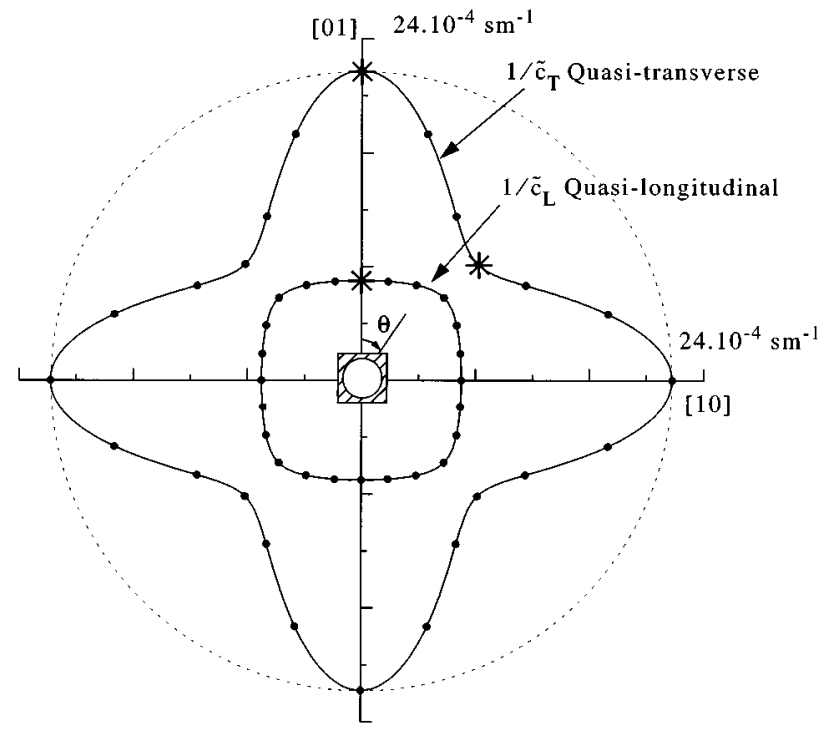

FIG. 9. Slowness curves for $P=0.503$ : Variations of the inverse of velocities as a function of the incident angle $\theta$. Full lines: semianalytical results. Points: FEM results. Stars: points for the calculation of the effective stiffness constants.

the three effective stiffness constants are then deduced:

$$
\begin{aligned}
& \tilde{c}_{66}=\tilde{\rho} \tilde{c}_{T}^{2}\left(0^{\circ}\right), \\
& \tilde{c}_{11}=\tilde{\rho} \tilde{c}_{L}^{2}\left(0^{\circ}\right), \\
& \tilde{c}_{12}=\tilde{\rho}\left(\tilde{c}_{L}^{2}\left(0^{\circ}\right)-2 \tilde{c}_{T}^{2}\left(45^{\circ}\right)\right) .
\end{aligned}
$$

With the help of Christoffel's equation, the longitudinal and transverse velocities can be calculated using effective properties, for any incident angle.

When $P=0.503$, Fig. 9 presents in polar coordinates the slowness curves for quasilongitudinal and quasitransverse waves, i.e., the variations of the inverse velocities as a function of the incident angle. The full lines correspond to the analytical results, using effective properties. The points correspond to the finite element method results. The stars correspond to the points chosen for the computation of the effective properties. The agreement is very good between the curves, validating the homogenization process.

Figure 10 presents the porosity variations of the normalized homogenized stiffness constants $\tilde{c}_{11} / c_{11}, \tilde{c}_{12} / c_{12}$, and $\tilde{c}_{66} / c_{66}$ where $c_{11}, c_{12}$, and $c_{66}$ are the PVC stiffness constants, and the anisotropic coefficient $A$ is defined as

$$
A=2 \tilde{c}_{66} /\left(\tilde{c}_{11}-\tilde{c}_{12}\right) \text {. }
$$

These curves show that the material is more anisotropic when the porosity increases.

\section{Tridimensional model of the periodic array of cylindrical pores}

The aim of this section is to evaluate the accuracy of the homogenization process with the help of experimental results. Three plates made of PVC are studied. They are all 20 $\mathrm{mm}$ thick, $309 \mathrm{~mm}$ long, and $206 \mathrm{~mm}$ wide. The first plate is full. The two other plates are periodically perforated with, respectively, 300 and 600 holes (Fig. 11). The hole's diameter is 

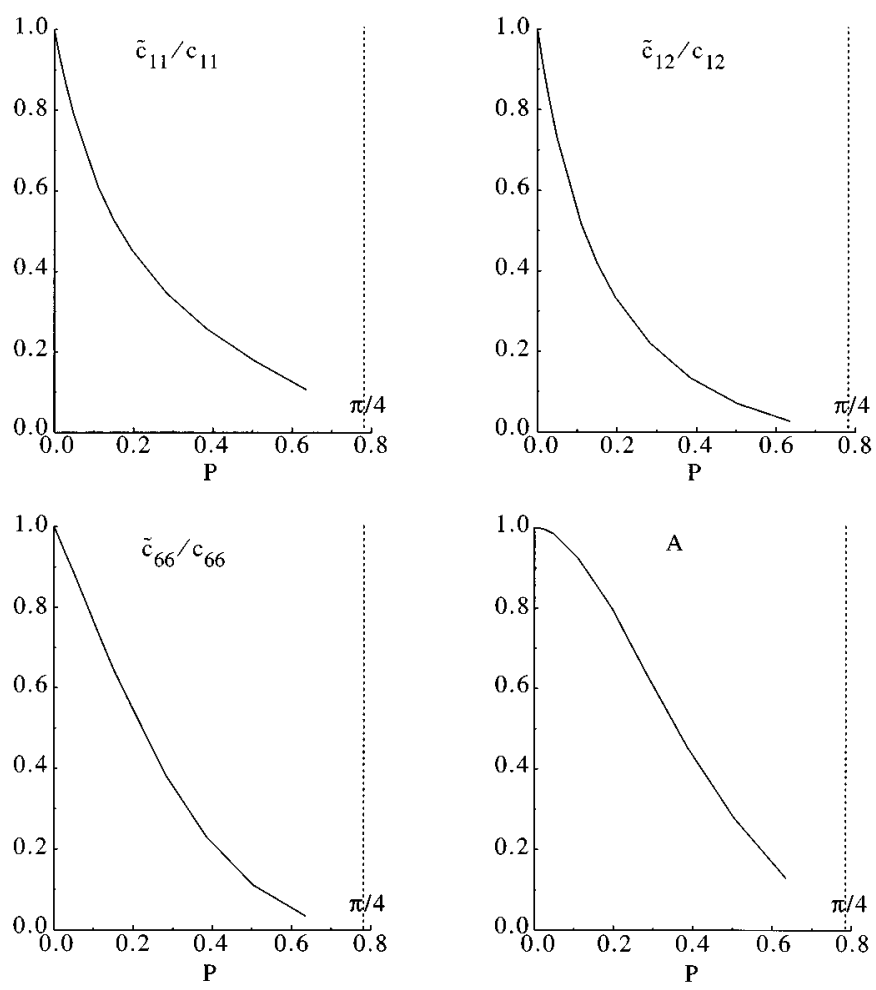

FIG. 10. Porosity variations of the normalized homogenized stiffness constants $\tilde{c}_{11} / c_{11}, \tilde{c}_{12} / c_{12}$, and $\tilde{c}_{66} / c_{66}$ and porosity variations of the anisotropic coefficient $A$.

equal to $7 \mathrm{~mm}$. Thus the porosities are, respectively, 0.192 and 0.385 for the second and the third plates.

First, the dispersion curves are calculated considering the three plates as infinite in the three space directions. For the full plate, a tridimensional unit cell is meshed, with arbitrary dimensions. For the two perforated plates, a tridimensional unit cell is meshed containing one pore of arbitrary thickness $2 h$ along the $z$ axis (Fig. 12). The wave vector is characterized by the two angles $\theta$ and $\varphi$ defined in Fig. 12 .

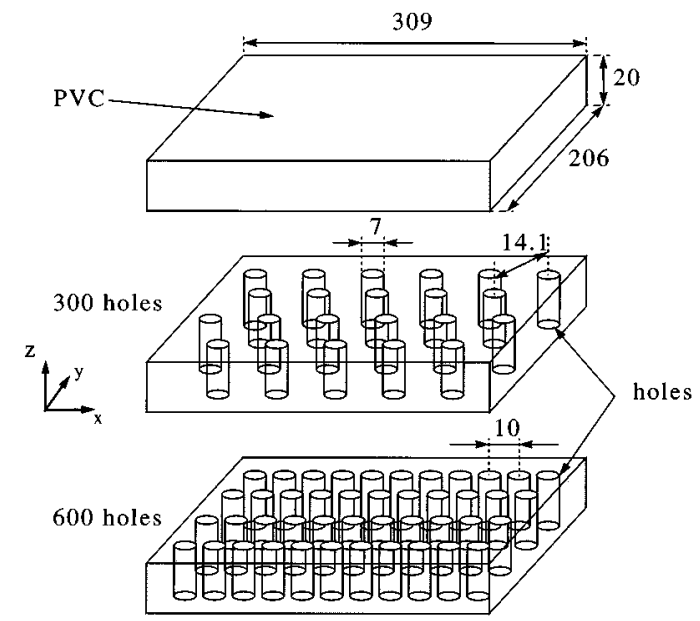

FIG. 11. Schematic description of the three plates made of PVC, without holes, containing 300 holes, and containing 600 holes.

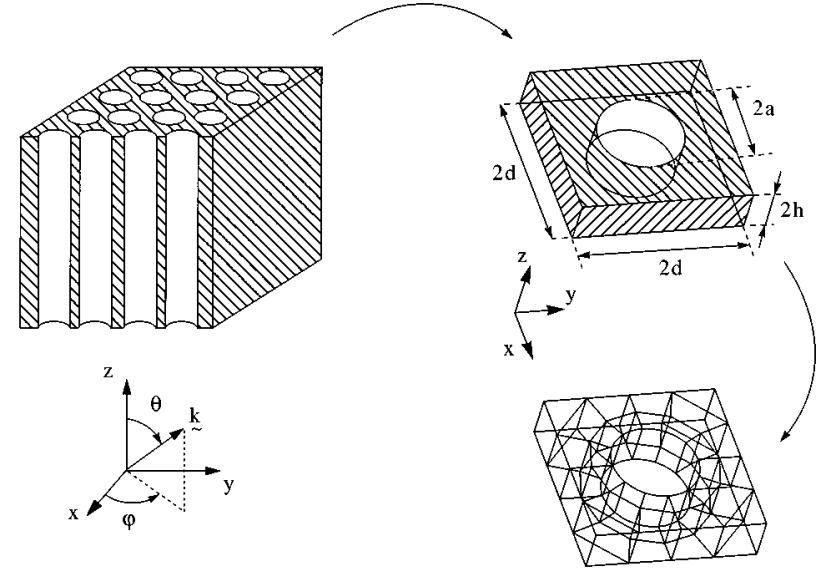

FIG. 12. Schematic description of a doubly periodic material, considered as a triply periodic material and finite element mesh of the unit cell.

The dispersion curves provide three low-frequency branches which can be approximated to three straight lines. Then, the two quasitransverse and the quasilongitudinal velocities are determined in the large wavelength limit.

In the same way, the material can be homogenized with the help of an anisotropic model, in which the stiffness tensor is

$$
[\tilde{c}]=\left[\begin{array}{cccccc}
\tilde{c}_{11} & \tilde{c}_{12} & \tilde{c}_{13} & 0 & 0 & 0 \\
\tilde{c}_{12} & \tilde{c}_{11} & \tilde{c}_{13} & 0 & 0 & 0 \\
\tilde{c}_{13} & \tilde{c}_{13} & \tilde{c}_{33} & 0 & 0 & 0 \\
0 & 0 & 0 & \tilde{c}_{44} & 0 & 0 \\
0 & 0 & 0 & 0 & \tilde{c}_{44} & 0 \\
0 & 0 & 0 & 0 & 0 & \tilde{c}_{66}
\end{array}\right] .
$$

In view of determining the six effective stiffness constants of the porous material in the large wavelength limit, the two quasitransverse and the quasilongitudinal velocities have to be computed for two different propagation directions $(\theta$ $\left.=90^{\circ}, \varphi=45^{\circ}\right)$ and $\left(\theta=45^{\circ}, \varphi=0^{\circ}\right)$. Then, using the classical Christoffel equation, the six effective stiffness constants are deduced.

Using the effective properties, the second and the third plates are meshed as an homogeneous and anisotropic plate. By this way, we avoid meshing the 300 or the 600 holes. Then, a modal analysis is performed which provides the resonance frequencies and the corresponding displacement field. The resonance modes are classified into four categories, according to the type of symmetry of their displacement

TABLE I. Resonance frequencies for the full plate made of PVC.

\begin{tabular}{|c|c|c|c|c|c|c|c|c|c|}
\hline No. & $\begin{array}{c}f_{\text {FEM }} \\
(\mathrm{Hz})\end{array}$ & Type & $\begin{array}{l}f_{\exp } \\
(\mathrm{Hz})\end{array}$ & $\begin{array}{c}\text { Error } \\
\%\end{array}$ & No. & $\begin{array}{c}f_{\mathrm{FEM}} \\
(\mathrm{Hz})\end{array}$ & Type & $\begin{array}{l}f_{\exp } \\
(\mathrm{Hz})\end{array}$ & $\begin{array}{c}\text { Error } \\
\%\end{array}$ \\
\hline 1 & 305.6 & $\mathrm{AA}$ & 307 & +0.5 & 6 & 1078.1 & SA & 1075 & -0.3 \\
\hline 2 & 346.3 & SS & 355 & +2.6 & 7 & 1290.4 & $\mathrm{AA}$ & 1257 & -2.6 \\
\hline 3 & 702.8 & AS & 701 & -0.3 & 8 & 1463.7 & SS & 1462 & -0.1 \\
\hline 4 & 815.0 & SS & 815 & +0.0 & 9 & 1873.1 & SS & 1867 & -0.3 \\
\hline 5 & 886.8 & SA & 902 & +1.7 & & & & & \\
\hline
\end{tabular}


TABLE II. Resonance frequencies for the plate made of PVC containing 300 holes.

\begin{tabular}{lccccccccc}
\hline \hline No. & $\begin{array}{c}f_{\text {FEM }} \\
(\mathrm{Hz})\end{array}$ & Type & $\begin{array}{c}f_{\text {exp }} \\
(\mathrm{Hz})\end{array}$ & $\begin{array}{c}\text { Error } \\
\%\end{array}$ & No. & $\begin{array}{c}f_{\text {FEM }} \\
(\mathrm{Hz})\end{array}$ & Type & $\begin{array}{c}f_{\text {exp }} \\
(\mathrm{Hz})\end{array}$ & $\begin{array}{c}\text { Error } \\
\%\end{array}$ \\
\hline 1 & 282.2 & AA & 272 & -3.6 & 6 & 919.4 & SA & 901 & -2.0 \\
2 & 288.6 & SS & 289 & +0.2 & 7 & 1134.8 & SA & 1116 & -1.6 \\
3 & 634.1 & AA & 621 & -2.1 & 8 & 1302.9 & SS & 1721 & -2.5 \\
4 & 680.2 & SS & 681 & +0.1 & 9 & 1577.3 & AA & 1548 & -1.9 \\
5 & 755.6 & AS & 748 & -1.0 & & & & & \\
\hline \hline
\end{tabular}

field with respect to the $x$ and $y$ axes: antisymmetricantisymmetric (AA), antisymmetric-symmetric (AS), symmetric-antisymmetric (SA), and symmetric-symmetric (SS). The experimental resonance frequencies are measured using an accelerometer applied on the plates excited by a harmonic sound.

Tables I-III present the first nine resonance frequencies for, respectively, the full plate, the 300-hole plate ( $P$ $=0.192)$, and the 600-hole plate $(P=0.385)$. The tables give the resonance frequencies calculated with the help of the finite element method, the types of the mode, the experimental frequencies, and the relative errors. The agreement is good between the experimental resonance frequencies and the calculated resonance frequencies, considering the plates as homogenous and anisotropic. When the porosity increases, the resonance frequencies decrease, particularly for the first and the third modes, for which the decrease is about $30 \%$. These tables give close results, validating the homogenization process. A better agreement could be obtained if the homogenization process took into account the effect of the air cavities, by meshing the pore as a fluid domain.

\section{CONCLUSION}

A model for predicting the propagation of plane acoustic waves in passive periodic materials has been presented and applied to the analysis of periodic materials containing different types of inclusions. It relies upon the finite element method, with the help of the ATILA code. It has allowed an easy identification of the propagation modes, with the help of the computed displacement fields. In the case of periodic array of pores, the porosity variations of the homogenized longitudinal and transverse velocities are presented, using the low-frequency branches of the curves. In the large wavelength limit, the homogenized properties of periodic porous material have been determined with the help of an aniso-

TABLE III. Resonance frequencies for the plate made of PVC containing 600 holes.

\begin{tabular}{lccccccccc}
\hline \hline & $\begin{array}{c}f_{\text {FEM }} \\
(\mathrm{Hz})\end{array}$ & Type & $\begin{array}{c}f_{\text {exp }}(\mathrm{Hz}) \\
\text { No. }\end{array}$ & $\begin{array}{c}\text { Error } \\
\%\end{array}$ & No. & $\begin{array}{c}f_{\text {FEM }} \\
(\mathrm{Hz})\end{array}$ & Type & $\begin{array}{c}f_{\exp } \\
(\mathrm{Hz})\end{array}$ & $\begin{array}{c}\text { Error } \\
\%\end{array}$ \\
\hline 1 & 192.2 & AA & 180 & -6.3 & 6 & 807.5 & SA & 772 & -4.4 \\
2 & 289.2 & SS & 273 & -5.5 & 7 & 956.1 & AA & 925 & -3.2 \\
3 & 480.5 & AS & 459 & -4.5 & 8 & 1027.3 & SS & 1005 & -2.2 \\
4 & 648.2 & SS & 626 & -3.5 & 9 & 1424.3 & SS & 1442 & +1.2 \\
5 & 721.9 & SA & 690 & -4.4 & & & & & \\
\hline \hline
\end{tabular}

tropic model. A careful validation has been carried out with periodically perforated plates, the resonance frequencies of which have been measured.

This technique can now be used for the analysis of the propagation of plane acoustic waves in single periodic materials, such as a waveguide, ${ }^{23,24}$ or in triply periodic materials, such as composite or periodic arrays of spherical inclusions. ${ }^{10}$ Moreover, an extension of the technique can be performed in a view to model coupled fluid-solid periodic materials ${ }^{2}$ and active periodic materials. ${ }^{14}$ In that case, the dispersion curves provide results of physical interest, particularly for piezocomposites: ${ }^{25-27}$ homogenized wave velocities, electromechanical coupling factors, depending on the poling axis of the ceramics. The homogenization process could be extended to these active periodic materials.

${ }^{1}$ L. Brillouin, Wave Propagation in Periodic Structures (Dover, New York, 1953).

${ }^{2}$ C. Audoly and G. Duméry, "Acoustic wave propagation in media containing two-dimensional periodically spaced elastic inclusions," in Physical Acoustics, edited by O. Leroy and M. A. Breazeale (Plenum, New York, 1991), pp. 219-224.

${ }^{3}$ R. Tao and P. Sheng, "First-principle approach to the calculation of elastic moduli for arbitrary periodic composites," J. Acoust. Soc. Am. 77, 16511658 (1985).

${ }^{4}$ B. A. Auld, Y. A. Shui, and Y. Wang, "Elastic wave propagation in threedimensional periodic composite materials," J. Phys. 45, 159-163 (1984).

${ }^{5}$ A. M. Braga and G. Herrmann, "Floquet waves in anisotropic periodically layered composites," J. Acoust. Soc. Am. 91, 1211-1227 (1992).

${ }^{6} \mathrm{~S}$. Nemat-Nasser and M. Yamada, "Harmonic waves in fiber-reinforced orthotropic elastic composites," J. Appl. Mech. 48, 967-971 (1981).

${ }^{7} \mathrm{~S}$. Baste and A. Gérard, "Etude des modes guidés dans les composites tri-dimensionnels," Rev. Acoust. 78, 43-48 (1986).

${ }^{8}$ J. D. Achenbach and M. Kitahara, "Harmonic waves in a solid with a periodic distribution of spherical cavities," J. Acoust. Soc. Am. 81, 595598 (1987).

${ }^{9}$ M. S. Bennett, "Free wave propagation in orthogonally stiffened cylinders," Ph.D. thesis, The University of Connecticut, Storrs, CT, 1989.

${ }^{10}$ A. A. Ruffa, "Acoustic wave propagation through periodic bubbly liquids," J. Acoust. Soc. Am. 91, 1-11 (1992).

${ }^{11}$ T. C. Ma, R. A. Scott, and W. H. Yang, "Harmonic wave propagation in an infinite elastic medium with a periodic array of cylindrical pores," J. Sound Vib. 71, 473-482 (1980).

${ }^{12}$ A. C. Hennion, R. Bossut, J. N. Decarpigny, and C. Audoly, "Analysis of the scattering of a plane acoustic wave by a periodic elastic structure using the finite element method: Application to compliant tube gratings," J. Acoust. Soc. Am. 87, 1861-1870 (1990).

${ }^{13}$ A. C. Hladky-Hennion and J. N. Decarpigny, "Analysis of the scattering of a plane acoustic wave by a doubly periodic structure using the finite element method: Application to Alberich anechoic coatings," J. Acoust. Soc. Am. 90, 3356-3367 (1991).

${ }^{14}$ A. C. Hladky-Hennion and J. N. Decarpigny, "Finite element modeling of active periodic structures: Application to 1-3 piezocomposites," J. Acoust. Soc. Am. 94, 621-635 (1993).

${ }^{15} \mathrm{~J}$. N. Decapigny, "Application de la méthode des éléments finis à l'étude de transducteurs piézoélectriques," thesis, Université des Sciences et Techniques de Lille, 1984.

${ }^{16}$ J. N. Decarpigny, J. C. Debus, P. Tierce, B. Tocquet, and D. Boucher, "In-air analysis of piezoelectric Tonpilz transducers in a wide frequency band using a mixed finite element-plane wave method," J. Acoust. Soc. Am. 78, 1499-1507 (1985).

${ }^{17}$ R. Bossut and J. N. Decarpigny, "Finite element modeling of radiating structures using dipolar damping elements," J. Acoust. Soc. Am. 86, 1234-1244 (1990).

${ }^{18}$ B. Hamonic, J. C. Debus, J. N. Decarpigny, D. Boucher, and B. Tocquet, "Analysis of radiating thin shell sonar transducers using the finite element method," J. Acoust. Soc. Am. 86, 1245-1253 (1990).

${ }^{19}$ B. Dubus, J. C. Debus, J. N. Decarpigny, and D. Boucher, "Analysis of mechanical limitations of high power piezoelectric transducers using finite element modeling," Ultrasonics 29, 201-207 (1991). 
${ }^{20}$ P. Langlet, "Analyse de la propagation des ondes acoustiques dans les matériaux périodiques à l'aide de la méthode des éléments finis," thesis, Université de Valenciennes et du Hainaut-Cambrésis, 1993.

${ }^{21}$ O. C. Zienkiewicz, The Finite Element Method (McGraw-Hill, New York, 1977).

${ }^{22}$ The $\lambda / 4$ criterion is related to the six- and eight-node isoparametric finite elements used in this work and due to the fact that the shape function variations are quadratic with respect to the coordinates along an element side. See, for example, Refs. 15-18.

${ }^{23}$ P. E. Lagasse, "Higher-order finite element analysis of topographic guides supporting elastic surface waves," J. Acoust. Soc. Am. 53, 1116-1122 (1973).
${ }^{24}$ M. Koshiba, S. Mitobe, and M. Suzuki, "Finite element solution of periodic waveguides for acoustic waves," IEEE Trans. Ultrason. Ferroelectr. 34, 472-477 (1987).

${ }^{25}$ M. Yamaguchi, K. Y. Hashimoto, and H. Makita, "Finite element method analysis of dispersion characteristics for 1-3 type piezoelectric composites," IEEE Ultrasonics Symposium, 1987, pp. 657-661.

${ }^{26}$ B. A. Auld and Y. Wang, "Acoustic wave vibrations in periodic composite plates," IEEE Ultrasonics Symposium, 1984, pp. 528-532.

${ }^{27}$ Y. Wang and B. A. Auld, "Numerical analysis of Bloch theory for acoustic wave propagation in one-dimensional periodic composites," IEEE Ultrasonics Symposium, 1986, pp. 261-264. 